\title{
A Review on Efficient Mac Layer Handoff Protocol to Reduce Handoff Latency for Wi-Fi Based Wireless Network
}

\author{
Mohini Joshi $^{{ }^{*}}$, Kishore Kumbhare ${ }^{2}$ \\ ${ }^{1}$ Department of Electronics and Communication, JIT, RGPV, Borawan, M.P (India) \\ ${ }^{2}$ Department of Electronics and Communication, JIT, RGPV, Borawan, M.P (India)
}

Available online at: www.isroset.org

Received: 02/Apr/2018, Revised: 08/Apr/2018, Accepted: 21/Apr/2018, Online: 30/Apr/ 2018

\begin{abstract}
WIFI-based wireless networks are becoming important components, because WIFI devices are widely used in laptops, PDAs and other mobile computing machines. In a WIFI-based wireless network, handoff management is a key service, as the radio range of the WIFI device is limited. Moreover, providing seamless roaming in wireless networks is mandatory for supporting real-time applications in a mobile environment, such as VoIP, online games, and e Conference. Recently, many solutions have been introduced to reduce MAC layer handoff latency; however, these solutions consider every mobile node separately, which would not be suitable for a large-scale environment. This paper proposes a novel MAC layer handoff protocol for large-scale WIFI-based wireless networks to support seamless real-time applications. In our scheme, before the mobile node starts initiating the MAC layer handoff process, it selects several neighboring nodes to help it scan available channels. All channels are divided into groups and scanned by these neighbors separately. Therefore, the number of scanning channels in each node is reduced, and scanning latency is minimized. According to simulation results in ns2, we conclude that our scheme can shorten MAC layer handoff latency greatly, and also achieve seamless handoff in terms of loss ratio of data packets.
\end{abstract}

Keywords:-IEEE 802. 11 Wlan Handoff, Access Point Handoff, Latency Selective, Scanning Neighbor Graph.

\section{INTRODUCTION}

IEEE 802.11 [1] protocol is the most widely used wireless connection standard in IP-based networks, and its advantages over other wireless connection protocols, such as low cost, easy deployment and high bandwidth, will make it an important component in next generation networks. However, the radio range in IEEE 802.11 is limited, and mobile nodes need to change access points (APs) frequently during the movement. Therefore, changing APs smoothly is the key issue in IEEE 802.11 networks. There are two phases in a handoff process: the MAC layer handoff and the network layer handoff. When the mobile node finds quality of signal, which can be measured by the received signal strength indication (RSSI) or a signal to noise ratio (SNR) below the predefined level in the MAC layer, the mobile node initiates the handoff process to find and associate with the AP that has the best quality of signal in the mobile node's neighborhood. After that, the new data routing path between the mobile node and its correspondent nodes should be re-established to maintain communication in the network layer. A comprehensive survey of existing handoff management solutions is given in [2]. IEEE $802.11 \mathrm{~b} / \mathrm{g}$ [1] protocol outlines the basic steps of the handoff process. Unfortunately, the original handoff latency is of several hundred milliseconds [3], while the requirement for real-time applications for MAC layer handoff latency is less than $50 \mathrm{~ms}$. Therefore, much research has proposed ways to reduce the handoff latency in recent years. However, these schemes do not work well in multichannel wireless networks, since the mobile node have to switch and scan multi channels. In IEEE $802.11 \mathrm{~b} / \mathrm{g}$, there are 14 channels, and channel $1-11$ are available for use in North America [1]. Because the average time to switch channels is $5 \mathrm{~ms}$ [4], the total switching time for 11 channels is more than $50 \mathrm{~ms}$, and it is hard to scan all channels with only one mobile node. Thus, reducing the number of scanning channels is an efficient way of minimizing handoff latency in MAC layer.

This paper presents an efficient MAC layer handoff protocol to reduce handoff latency in IEEE 802.11-based wireless networks. When a handoff process is initiated, the mobile node requires nodes in a certain range to help it scan the available channels. These nodes construct a temporary group. The scanning channels are distributed to nodes in the group; in other words, each node only needs to scan some of the channels. Therefore, the whole probing process is divided into small parts and all of these parts can be executed simultaneously. Moreover, the scanning result, which can be used as a handoff target during a certain period of time, is shared by the group members. The nodes can then restore the result in the cache, since moving nodes in the group might 
associate with the same AP soon. Using this scheme, the probing load is distributed and handoff latency can be minimized especially in large-scale networks.

\section{Objective of Study}

The objectives that is to be achieving using new algorithm is:-

1) To Improve Handoff Latency with respect to Active / Passive scanning.

2) To improve with respect to Active / Passive scanning, during handoff in Wi-Fi based Wireless Network.

3) To develop a solution that has more generalized applicability.

4) To develop a solution that requires no additional hardware.

\section{Issue of old Article}

Akyildiz, J. Xie, and S. Mohanty[2] In this article we give a comprehensive survey of mobility management techniques in NG all-IP based wireless systems. Since global roaming in heterogeneous wireless environments will become an essential trend in the near future, we focus on mobility management techniques in heterogeneous wireless networks. We describe current proposed protocols for mobility management in NG all-IP-based wireless systems.

Arunesh Mishra and Minho Shin[3] We find that the sequence of messages Exchanged during the handoff process can also differ with the STA being used. One of the more interesting results of our work is that current WLAN equipment will not meet the expectations (replacing or augment $4 \mathrm{G}$ systems) that many have. This is because the handoff latencies we measured far exceed guide lines for jitter in voice over IP (VoIP) applications where the overall latency is recommended not to exceed $50 \mathrm{~ms}$

Chintala and Q.-A. Zeng[4] The handoff delay perceived in the existing IEEE 802.11 WLANs is well above the delay constraints of multimedia applications like VoIP. Two novel schemes to reduce handoff latency by using inter-AP communication were proposed in this paper. The first scheme proposed avoided probe wait delay by receiving all the probe responses from the old AP. Though the handoff latency is decreased considerably, this scheme does not meet the recommended multimedia restrictions. Another scheme has been presented which spreads out the total handoff latency and interleaves normal data transmission with channel scan. This scheme can meet the VoIP constraints.

Velayos and G. Karlsson[5] The handoff process was split into three sequential phases: detection, search and execution. We studied the detection based on failed frames (link-layer detection) instead of weak signal because it produces less handoff events. We have shown that the linklayer detection phase can be reduced to three consecutive non-acknowledged frames when stations are transmitting. In the same conditions we used during our measurements, this time would be around $3 \mathrm{~ms}$, which is approximately 300 times shorter than the fastest measured detection phase. When stations are only receiving, we identified the beacon interval as the key factor in reducing detection time. Our simulations suggest $60 \mathrm{~ms}$ as an adequate beacon interval. We have also shown that using active scanning with its timers Min Channel Time and Max Channel Time set to $1 \mathrm{~ms}$ and $10.24 \mathrm{~ms}$ respectively can reduce the search phase by $20 \%$ compared to the shortest measured one. Finally, the execution phase can be reduced with pre-authentication, but our measurements indicate that it is a very short phase and its reduction will not significantly decrease the total handoff time when using the current authentication methods.

\section{Modeling}

In this section, our improvement on the original IEEE 802.11 MAC layer handoff process is proposed. The basic idea is that instead of scanning all available channels by the mobile node to look for the new AP, channels are divided into different groups, and each group is assigned to an assistant node. These assistant nodes, which are selected according to their distances from the mobile node, are responsible for helping the mobile node to scan channels. With this scheme, the workload of scanning channels are distributed, and only some of the available channels, not all of them, are scanned by each assigned node. Thus, it takes less time to find the appropriate channel, and consequently the handoff latency can be minimized .In wireless networks, the mobile node can measure the quality of the signal strength by SNR or RSS. We use RSS in our scheme. We predefine three Received Signal Strength (RSS) thresholds: $R S S_{l}, R S S_{h}$ and $R S S_{g} . R S S_{l}$ is the threshold used to trigger handoff, while $R S S_{h}$ is used to construct a temporary handoff group consisting of assistant nodes. $R S S_{h}$ is a little higher than $R S S_{l}$, so that it ensures there are assistant nodes before the mobile node starts handoff. $R S S_{g}$ is used to define the largest distance from the mobile node and its neighbor nodes, since the RSS decreases as the distance increases. Only the neighbor nodes that are close to the mobile node are selected as assistant nodes. This scheme is used to ensure a tolerant error range when we use the RSS detected by the neighbor nodes as the RSS detected by the mobile node.

\section{A. Group Construction Scheme}

When the current RSS $\left(R S S_{\text {curr }}\right)$ is lower than $R S S_{h}$, the grouping process is triggered. The mobile node then broadcasts a grouping request message with a predefined RSS $R S S_{g}$ within its neighborhood. Upon receiving this grouping request message, the neighbor node gets the RSS and tests whether the RSS is greater than $R S S_{g}$. If the RSS is 
less than $R S S_{g}$, the request message is ignored; otherwise, the neighbor node that want to participate in the distributed probing process should reply to the mobile node with a grouping response message. Next, the mobile node collects all of the grouping response messages, and assesses the response nodes as its scanning assistant nodes, which will be used in next step. Figure 2 shows an example of our scheme. In this case, mobile node $\mathrm{N}$ considers neighbor nodes in range $r 1$ as the assistant nodes; therefore, nodes $0,1,2,3$ and 4 are selected. Later, when the mobile node finds that the current RSS $\left(R S S_{\text {curr }}\right)$ is lower than $R S S_{l}$, it decides to do the handoff. First, it divides the channels into different groups according to the number of scanning assistant nodes, and assigns one group to one assistant node. The procedure for assigning channels to assistant nodes is shown in Algorithm 1. Considering the example shown in Figure 2, if we suppose that the current working channel of the mobile node is channel 1, and only 11 channels are used; then channels 2 and 7 are assigned to node 0 , channels 3 and 8 are assigned to node 1 , channels 4 and 9 are assigned to node 2 , channels 5 and 10 are assigned to node 3 and channels 6 and 11 are assigned to node 4 .

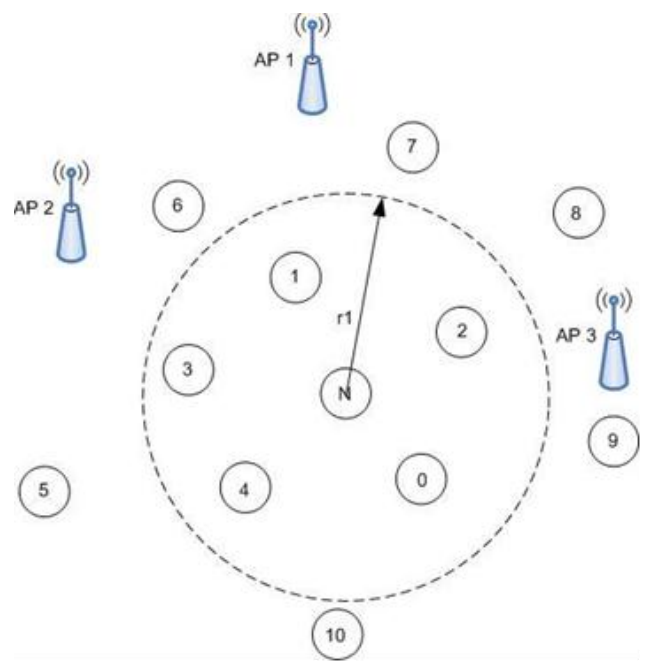

Fig.1. An example of our scheme

\section{B. Distributed Scan Mechanism}

After different groups of nodes have been constructed, the mobile node broadcasts the grouping confirmation message to all of the scanning assistant nodes, in which the scanning channels of each assistant node are encapsulated. Upon receiling the grouping confirmation message, the assistant node knows which channels it needs to scan. The assistant node then switches to the channel that is going to be scanned, broadcasts a probe request message, waits for probe response messages, and switches to the next channel assigned to collect available APs' information. After all of the assigned channels have been scanned, the assistant node selects the

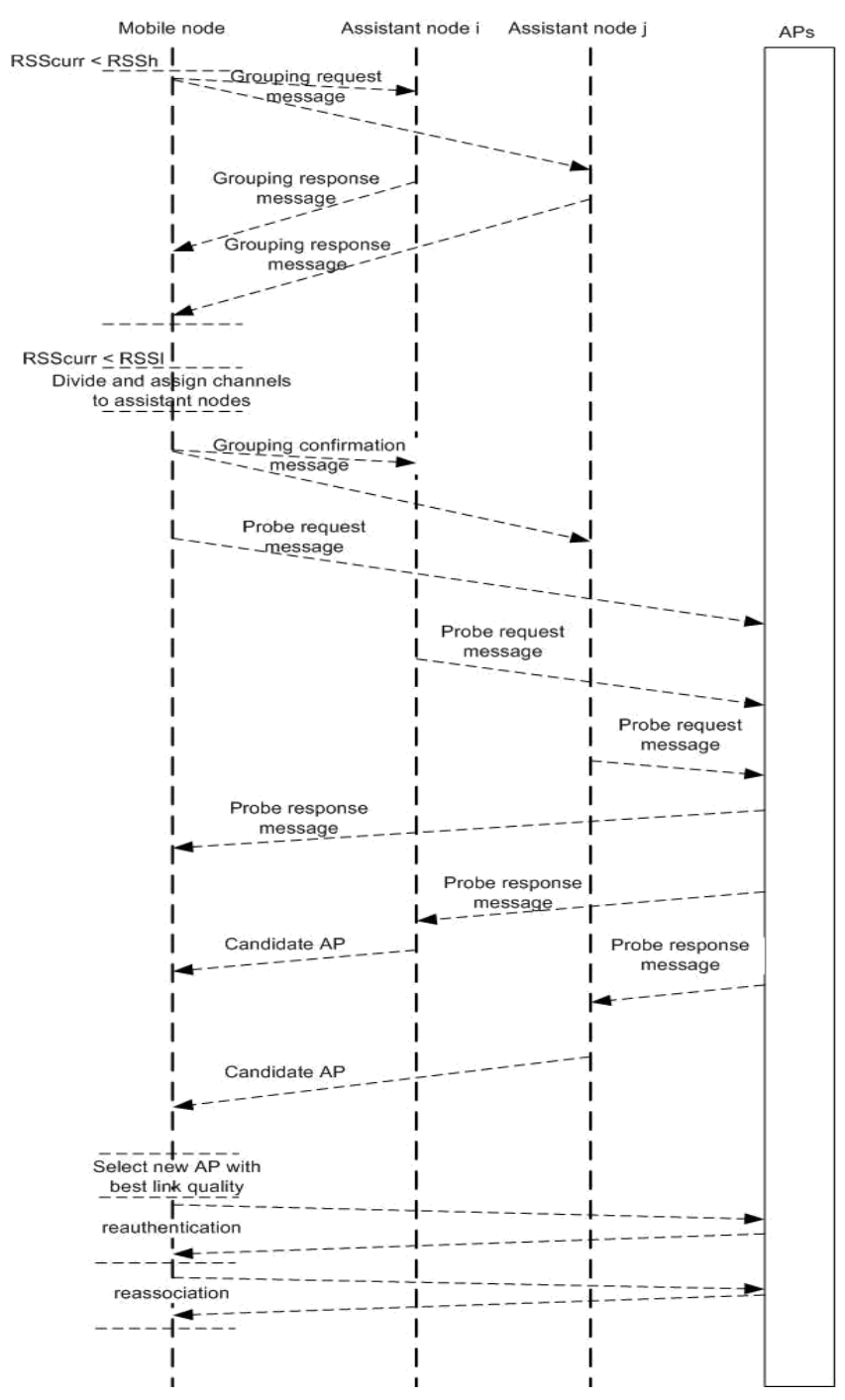

Fig. 2. Sequence diagram of our MAC layer handoff scheme with distributed scanning

AP with the best signal quality as the candidate AP, and responds to the mobile node with the information of this candidate AP. When the mobile node receives all return messages from assistant nodes, it selects as the new AP the AP with the best signal quality of all candidates. In Figure 1, the mobile node selects AP3 as the new AP. After authentication and association with the new AP, the MAC layer handoff process is completed and the network layer handoff is initiated in order to re-established logical connection with correspondent nodes. The whole procedure for our MAC layer handoff scheme with distributed scanning is shown in Figure 2. 


\section{Caching Scheme}

A caching structure is used to store AP information based on the result of the distributed probing, since we find that all assistant nodes are close to the mobile node, and that it is also possible for them to re-associate with the same AP as the mobile node after a short period. When the mobile node collects all of the scanning results from assistant nodes, it broadcasts a message with the new AP to all assistant nodes. The assistant node will save the new AP in its caching structure with a lease time $T_{\text {lease }}$. Only the latest AP is saved in the cache, and the old AP is usually overwritten. During $T_{\text {lease }}$, when the assistant node wants to start handoff, it will first try to re-authenticate with the AP in the cache. If the AP accepts the assistant node, the assistant node can re-associate with the AP directly and the scanning time is waived; otherwise, the assistant node should start a complete distributed handoff process. The flowchart of handoff using caching scheme is shown in Figure 3

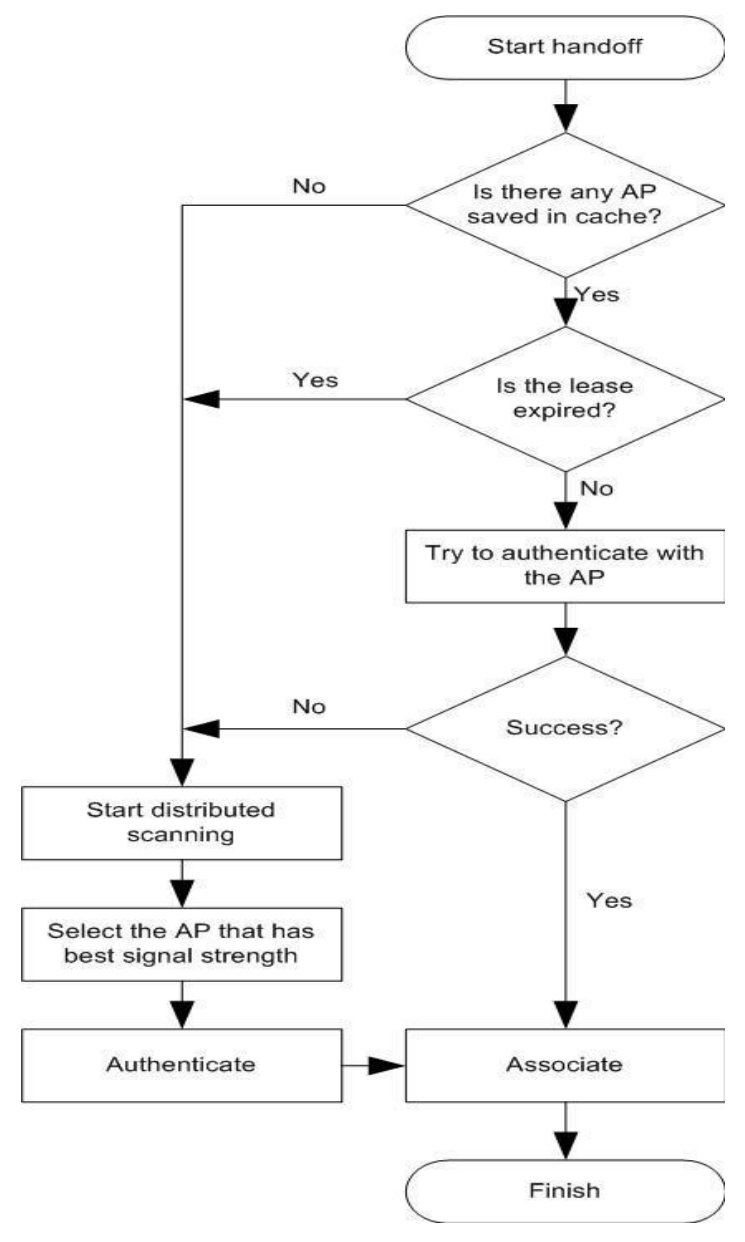

Fig. 3. Flowchart of handoff using the caching scheme

\section{Conclusion}

Handoff management plays an important role in seam-less wireless networks. This paper proposes a novel MAC layer handoff scheme that uses distributed probe to minimize handoff latency. Instead of scanning all channels with the mobile node, channels are divided and assigned to several assistant nodes that are close to the mobile node. This scheme allows each node to scan only a few channels; resulting in a distributed probing process. Moreover, a caching scheme is designed to further decrease handoff latency. Simulation results show that handoff latency can be reduced to $50 \mathrm{~ms}$ or less, which is required by real-time applications even in a large-scale wireless network. Thus, our handoff scheme can be used to support seamless roaming in wireless networks, which support real-time applications such as VoIP, eConference, and so on. For the future work, the actual implementation is going to be realized to further support our handoff scheme.

\section{References}

[1] "IEEE standard for information technologytelecommunications and information exchange between systems-local and metropolitan area networks-specific requirements - part 11: Wireless lan medium access control (mac) and physical layer (phy) specifications," http://standards.ieee.org/getieee802/802.11.html, 2007.

[2] I. F. Akyildiz, J. Xie, and S. Mohanty, "A survey of mobility manage-ment in next-generation all-IP-based wireless systems," IEEE Wireless Communications, vol. 11, no. 4, pp. 16-28, August 2004.

[3] W. A. Arunesh Mishra, Minho Shin, "An empirical analysis of the ieee 802.11 mac layer handoff process," ACM SIGCOMM Computer Communication Review, vol. 33, no. 2, pp. 93-102, April 2003.

[4] V. M. Chintala and Q.-A. Zeng, "Novel mac layer handoff schemes for ieee 802.11 wireless lans," in IEEE Wireless Communications and Networking Conference, March 2007, pp. 4435-4440.

[5] H. Velayos and G. Karlsson, "Techniques to reduce the ieee 802.11 b handoff time," in 2004 IEEE International Conference on Communica-tions, vol. 7, June 2004, pp. 3844-3848.

[6] M. Shin, A. Mishra, and W. A. Arbaugh, "Improving the latency of

802.11 hand-offs using neighbor graphs," in Proceedings of the 2nd international conference on Mobile systems, applications, and services, June 2004, 
pp. 19-26.

[7] S. Shin, A. G. Forte, A. S. Rawat, and H. Schulzrinne, "Reducing mac layer handoff latency in ieee 802.11 wireless lans," in Proceedings of the second international workshop on Mobility management and wireless access protocols, October 2004, pp. 70-83.

[8] N. Mustafa, W. Mahmood, A. A. Chaudhry, and M. Ibrahim, "Pre-scanning and dynamic caching for fast handoff at mac layer in ieee

802.11 wireless lans," in IEEE International Conference on Mobile Adhoc and Sensor Systems Conference, November 2005, pp. 1-8.

[9] K. Ramachandran, S. Rangarajan, and J. C. Lin, "Makebefore-break mac layer handoff in 802.11 wireless networks," in 2006 IEEE ICC, vol. 10, June 2006, pp. 4818-4823.

[10] Y. Liao and L. Cao, "Practical schemes for smooth MAC layer handoff in 802.11 wireless networks," in WoWMoM, June 2006, pp. 1-10.

[11]I. Ramani and S. Savage, "SyncScan: practical fast handoff for 802.11 infrastructure networks," in Proceeding of IEEE INFOCOM, vol. 1, March 2005, pp. 13-17.

[12] "The network simulator - ns-2," http://www.isi.edu/nsnam/ns/. 